\title{
3 \\ Apuesta por el aprendizaje significativo desde procesos asincrónicos: algunas acciones para mejorar la equidad educativa a raíz de la COVID-19
}

\section{Commitment to meaningful learning via asynchronous processes: some actions to improve educational equity as a result of Covid-19}

\section{Compromisso com a aprendizagem significativa a partir de processos assíncronos: algumas ações para melhorar a equidade educacional como resultado da Covid-19}

Juan Pablo Escobar Galo ${ }^{1}$

«No hay inmovilismo en la historia. Siempre hay algo que podemos hacer y rehacer» Freire (2008, p. 37)

\section{Resumen}

La Pandemia causada por la COVID-19 provocó que muchos de los procesos educativos que se desarrollaban de forma presencial fueran continuados, de forma inmediata, por medio de la

\section{Recibido: 9-7-2021 - Aceptado: 5-10-2021}

1 Guatemalteco humanista en búsqueda de un mundo más digno y justo para todas y todos. Doctor en Educación, postgrado en Especialización del Aprendizaje y Docencia Universitaria, magíster en Filosofía, licenciado en Administración Educativa y profesor de Enseñanza media en Pedagogía y Ciencias de la educación. Actualmente es decano y catedrático de la Universidad Rafael Landívar de Guatemala.

(D) https://orcid.org/0000-0001-5546-1953. Correo electrónico: jescobar@url.edu.gt 
virtualidad informática, siendo estos implementados, en su mayoría, a través de actividades sincrónicas que buscaban simular el aula presencial. En muchos lugares urbanos dicha migración era posible pero no en las poblaciones que tienen poco acceso a internet, sobre todo en el interior del país. El presente ensayo presenta dos herramientas que responden a la atención de las y los estudiantes que sufren las consecuencias de la brecha digital para continuar con regularidad sus estudios, pero que necesitan que las instituciones educativas respondan de forma creativa y efectiva para lograr continuar de forma regular con su formación profesional en el ámbito universitario. La apuesta del presente texto es mostrar cómo los procesos asincrónicos, bien planificados y orientados, pueden responder a las necesidades ya descritas y garantizar el aprendizaje significativo. También se desea exponer, como el uso del WhatsApp puede ser mediado por procesos pedagógicos para que se utilice con fines educativos de forma sincrónica o asincrónica.

Palabras clave: brecha digital, educación asincrónica, educación superior y WhatsApp como herramienta educativa, equidad educativa.

\begin{abstract}
The Pandemic caused by Covid-19 caused many of the educational processes that were developed in person to be continued, immediately, through computer virtuality, most of them being implemented through synchronous activities that sought to simulate the in-person classroom. In many urban places such migration was possible but not in populations that have little access to the internet, especially in the interior of the country. This essay presents two tools that respond to the attention of students who suffer the consequences of the digital divide to continue their studies regularly, but who need educational institutions to respond creatively and effectively in order to continue, in a regular manner, with their professional training at the university level. The aim of this text is to show how asynchronous processes, well planned and oriented, can respond to the needs already described and guarantee meaningful learning. It is also desired to expose how the use of WhatsApp can be pedagogically mediated so that it is used for educational purposes synchronously or asynchronously.
\end{abstract}

Keywords: asynchronous education, digital divide, educational equity, higher education and WhatsApp as an educational tool.

\title{
Resumo
}

A Pandemia provocada pela Covid-19 fez com que muitos dos processos educativos que se desenvolviam presencialmente fossem continuados, de imediato, através da virtualidade informática, sendo estes implementados principalmente através de atividades síncronas que procuravam simular a sala de aula. Em muitos lugares urbanos, essa migração foi possível, mas não em populações com pouco acesso à internet, especialmente no interior do país.

Este ensaio apresenta duas ferramentas que respondem à atenção dos alunos que sofrem as consequências da exclusão digital para continuar os seus estudos regularmente, mas que precisam que instituições de ensino que respondam de forma criativa e eficaz para poderem continuar de forma regular no ambiente universitário. O objetivo deste texto é mostrar como processos 
assíncronos, bem planejados e orientados, podem responder às necessidades já descritas e garantir uma aprendizagem significativa. Também se deseja expor como o uso do WhatsApp pode ser mediado pedagogicamente para que seja utilizado para fins educacionais de forma síncrona ou assíncrona.

Palavras-chave: educação assíncrona, exclusão digital, equidade educacional, ensino superior e WhatsApp como ferramenta educacional.

\section{Introducción}

El presente ensayo tiene como principal finalidad compartir algunas experiencias que han ayudado en la época de pandemia, causada por la COVID-19, a dar continuidad a los procesos de aprendizaje de las y los estudiantes universitarios del interior del país de una universidad privada de Guatemala, quienes no gozan de un buen acceso a internet, lo cual ha obligado a crear alternativas que favorezcan el aprendizaje desde acciones asincrónicas por medio de guías de aprendizaje y haciendo uso del WhatsApp como herramienta educativa.

En un primer momento se presentan algunos datos que permiten contextualizar la situación de la población estudiantil y lo que implica la brecha digital. Seguido al punto anterior, se muestran dos herramientas educativas que pueden ser de utilidad para cualquier educador y educadora, para acompañar a sus estudiantes durante la pandemia y luego de ella.

Y para finalizar, a manera de conclusión, se evidencia que es posible llevar a cabo acciones concretas que permitan garantizar el aprendizaje autónomo de quienes no gozan de buena conectividad digital pero que también deben recibir una educación de calidad como derecho humano.

\section{Contextualización}

El último censo poblacional realizado por el Estado de Guatemala en el 2018, reflejó que, en términos de conexiones y servicios básicos las guatemaltecas y los guatemaltecos tienen un $58.9 \%$ de acceso a tubería de red de agua dentro de la vivienda, el $88.1 \%$ de la población cuenta con energía eléctrica y el $55.6 \%$ tiene servicios sanitarios idóneos para la vida en el hogar, lo cual evidencia que muchas comunidades, sobre todo del interior del país, no gozan aún de los servicios básicos en sus viviendas, por citar algunos datos, evidenciando, desde ya, la imposibilidad de tener acceso a internet, al no contar con las condiciones mínimas para el desarrollo de una vida digna en su hogar. Aunque hay diversos estudios sobre el aumento del consumo de internet en la población guatemalteca, la Agencia EFE (2019) afirma que el $62 \%$ de las personas censadas utilizan un teléfono móvil pero 
que, solo el $29.3 \%$ cuenta con internet residencial, lo cual revela la desigualdad de acceso a la educación virtual vinculada con el uso de internet.

La educación virtual servida a través de procesos informáticos sincrónicos ha resultado, en la mayoría de los casos, la primera opción para continuar con los procesos formativos formales, tanto en el nivel escolar como en la educación superior, sin tomar en cuenta los datos ahora compartidos. Es decir, el aula presencial ha sido "replicada" en el aula virtual, intentando, incluso, cumplir con los mismos horarios, técnicas y metodologías educativas y al mantener los procesos de evaluación del aprendizaje muy similares a los utilizados en las clases presenciales.

Por su parte Giannini y Grant (2020), mencionan que un reto urgente de atender, por la migración de gran cantidad de procesos educativos al modelo virtual, es lograr reducir la brecha digital como estrategia que garantice la equidad educativa, lo cual debe pasar por el análisis de aspectos como el acceso a internet, la formación de las educadoras y los educadores y la comunicación entre los centros educativos y las familias. Dicho reto se vuelve aún más complejo cuando los lugares en donde habitan las y los estudiantes, y en donde ahora están confinados al "resguardo" de la pandemia, no gozan de las condiciones e infraestructura necesaria para el acceso a la educación virtual informatizada. Esto demanda pensar en una apuesta educativa que no favorezca solo procesos virtuales o a distancia sincrónicos, por medio del uso exclusivo de internet, sino también el desarrollo de actividades asincrónicas que, de forma creativa logren acompañar los procesos de aprendizaje de los niños, las niñas, y las y los jóvenes que están confinados, o al menos, sin llegar a la escuela o a la universidad.

Es bajo dicha línea de reflexión, desde lo asincrónico, que se hace necesario fortalecer los procesos de aprendizaje-enseñanza autónomos, al crear guías de aprendizaje que garanticen el éxito educativo de la población que no tiene o tiene muy poco acceso a internet y que se pueden apoyar en el uso del teléfono celular y, desde casa, trabajar de forma remota y enviar sus guías de aprendizaje resueltas a través de WhatsApp, para tener, incluso, la posibilidad de mantener una comunicación sincrónica con sus docentes, compañeras y compañeros.

A criterio del Departamento de Educación de la Universidad Rafael Landívar de Guatemala (2020, p. 6):

El trabajo autónomo es una actividad estratégica, mediante la cual se pretende que el estudiante sea capaz de tomar decisiones sobre su propio aprendizaje; en este sentido, el docente se convierte en un facilitador que propone actividades para el desarrollo de habilidades de pensamiento de los estudiantes, tales como 
leer comprensivamente, relacionar, investigar, contrastar información, escribir coherentemente, desarrollar capacidad de búsqueda de información y aplicación de aprendizajes, llevándolo poco a poco a descubrir cómo aprende mejor. La función del docente, entonces, es la de ser un planificador de este trabajo; se convierte en un acompañante a distancia, en la virtualidad.

Desde dicha concepción educativa, no es indispensable el uso del internet de forma permanente para garantizar un aprendizaje significativo, sino que son las actividades y las estrategias metodológicas utilizadas, junto con el esfuerzo intelectual de las profesoras y los profesores y de las alumnas y los alumnos, las que favorecen el aprendizaje, más allá de la conexión o no a internet. Bajo esta lógica, en este espacio no se está apelando a que el internet llegue a todos los rincones del país, lo cual debe ser atendido a mediano o corto plazo por el Estado de Guatemala, porque se tiene consciencia de que esa no es una realidad viable ahora por múltiples factores y no será discutido en este ensayo. Lo que desea la actual reflexión es compartir propuestas reales y viables para que los procesos de aprendizaje de aquellas y aquellos que son víctimas de la brecha digital tengan acceso a la educación y puedan continuar de forma satisfactoria sus estudios. Las acciones que aquí se comparten han sido contempladas para procesos de aprendizaje en la educación superior, pero de igual manera pueden ser implementadas en diferentes niveles educativos.

Una forma práctica y sencilla de garantizar el aprendizaje significativo, la cual no requiere de mayor complejidad informática es la elaboración de guías asincrónicas de aprendizaje. Quizás elaborar guías de aprendizaje puede parecer algo poco novedoso y bastante común para varias educadoras y varios educadores. Sin embargo, en educación, por el uso exagerado de las Tic o por la implementación de metodologías "innovadoras" en muchas ocasiones se ha olvidado el desarrollo de acciones sencillas pero efectivas, que bien planificadas y con objetivos claros darán buenos resultados. Dichas guías, al ser estructuradas de manera correcta y en miras de favorecer el aprendizaje autónomo de las y los estudiantes, el cual ya ha sido descrito en la cita anterior, pueden responder a los problemas de conectividad a internet y así, garantizar el aprendizaje significativo de las y los estudiantes. Esto requiere reforzar en el profesorado los procesos que deben contemplar en la elaboración de estas, teniendo en cuenta los elementos mostrados en la gráfica que se comparte a continuación. 


\section{Gráfico 1}

Propuesta del proceso de aprendizaje

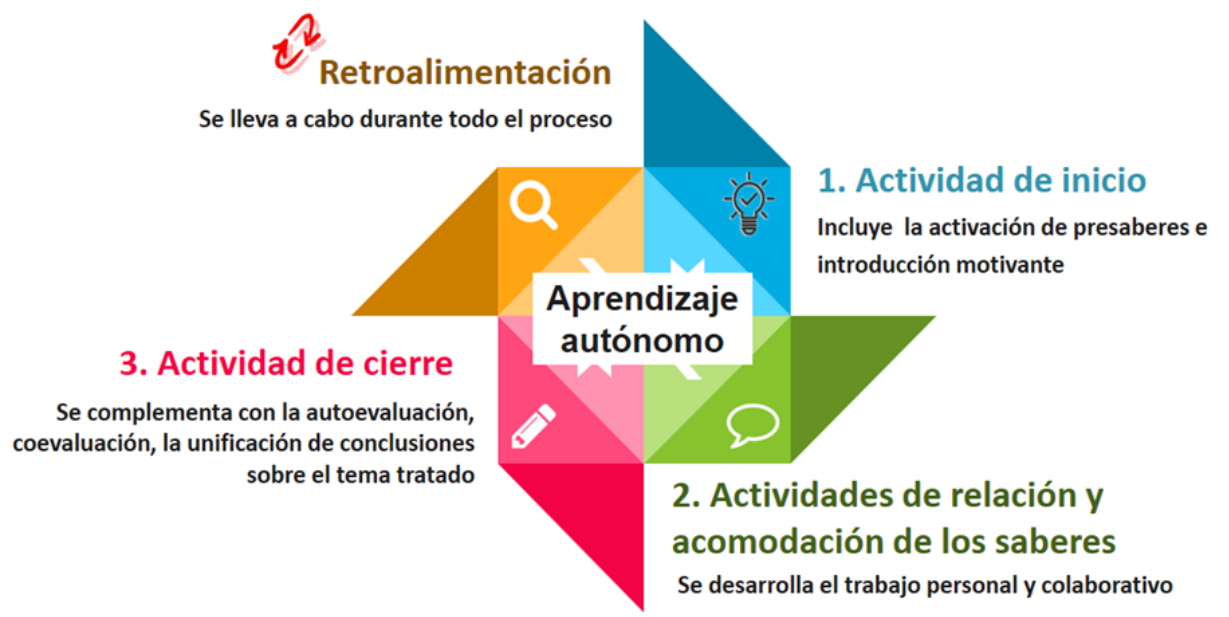

Nota. Tomado de: Departamento de Educación (2020), Imagen N. ${ }^{\circ}$ 1. Propuesta del proceso de aprendizaje.

Frente al confinamiento en los hogares, a causa de la COVID-19, muchas y muchos estudiantes, en su mayoría del interior del país y de áreas rurales, no estaban logrando continuar con sus estudios de forma exitosa, pero con el deseo de garantizar un aprendizaje significativo para todas y todos y, con el ánimo de mitigar la brecha digital que mantenía a las mismas y a los mismos en riesgo de no continuar con sus estudios superiores, a continuación se detallan, de forma breve, algunas acciones que se realizaron desde una propuesta asincrónica, por parte de la Universidad Rafael Landívar de Guatemala, con el deseo de compartir algunas propuestas que han sido viables y exitosas para lograr el aprendizaje de las y los estudiantes y que, con facilidad puede ser implementadas en otras instituciones educativas.

En primer lugar, durante la última quincena del mes de marzo del 2020, en respuesta inmediata al confinamiento y a las restricciones gubernamentales que surgieron fruto de la pandemia, se realizó un diagnóstico en todos los campus y sedes en donde la universidad tiene presencia, sobre el acceso a internet de las y los estudiantes y de las y los docentes. El diagnóstico reveló que una población significativa, mayormente de estudiantes, tenía poco acceso a internet y algunos muy poco, sobre todo por medio de computadoras, aunque un buen número de ellas y ellos lograban tener acceso a través del teléfono celular. 
En respuesta al diagnóstico, durante el mes de abril del mismo año, se implementó un plan piloto en base a los datos obtenidos, orientado a la sistematización de experiencias asincrónicas exitosas en el Campus de Quiché, ubicado a 159.7 km de la ciudad capital, en la región noroccidental del país, y en la Sede de la Antigua, ubicada a $46 \mathrm{~km}$ al oeste ciudad capital de la República. Dentro de sus hallazgos, el plan piloto implementado reveló que el trabajo asincrónico era el que más beneficios educativos brindaba a las y los estudiantes en época de confinamiento y la herramienta más utilizada y efectiva era el uso del WhatsApp.

A raíz de los resultados del plan piloto ya descrito, se llevaron a cabo múltiples capacitaciones dirigidas a docentes y coordinadores académicos y coordinadoras académicas en torno a la elaboración de guías para el desarrollo del aprendizaje autónomo y sobre el uso del WhatsApp como herramienta de apoyo educativo. Además, de forma conjunta al desarrollo de las capacitaciones, se crearon dos herramientas que fueron puestas al servicio de toda la comunidad educativa y que se describen a continuación.

\section{Guía de aprendizaje asincrónico}

La primera herramienta creada fue un modelo de Guía de aprendizaje asincrónico, la cual consta de los siguientes elementos: los datos generales del curso, los objetivos de aprendizaje, los productos a entregar, el material de apoyo, los pasos o los procesos para lograr los objetivos, el medio de entrega, la fecha de entrega, el punteo o la valoración y la rúbrica o los criterios de evaluación.

La Guía de aprendizaje asincrónico se comparte a partir del siguiente párrafo, la cual como ya se ha mencionado, con anterioridad, no es algo que no realizaran ya viarias educadoras y varios educadores, incluso la duda podría ser ¿qué de novedoso tiene la misma?, y la respuesta es: que luego de realizar el plan piloto, de dialogar con las y los estudiantes y con las educadoras y los educadores y de consultar con algunas expertas y algunos expertos en el tema educativo, era necesario crear un instrumento que ayudara a sistematizar, orientar y estructurar las acciones educativas que debían desarrollar las y los estudiantes desde casa, tomando en cuenta orientaciones sencillas, claras, prácticas y viables. A continuación, se comparte el modelo creado y utilizado por Escobar-Galo y Ambrosy (2020), el cual sirvió de base para las capacitaciones docentes. Es importante resaltar que algunos aspectos de la guía que se presenta después han sido ampliados o actualizados por el autor en relación con la propuesta original, pero sin alterar su esencia. 


\section{Guía de actividad de aprendizaje asincrónico o a distancia}

\section{Datos generales del curso:}

- $\quad$ Nombre del curso.

- Datos del (la) profesor (a): nombre, correo electrónico, teléfono celular, otros.

- Datos del (los/las) estudiante (s) enlace: correo electrónico, teléfono celular, otros.

- Datos de la coordinadora facultativa o del coordinador facultativo: correo electrónico, teléfono celular, otros.

- Título o nombre de la guía de aprendizaje.

\section{Objetivos de aprendizaje:}

Detallar los objetivos de aprendizaje que la guía de trabajo desea formar. Es bueno recordar que los objetivos de aprendizaje definen los procesos cognitivos, habilidades, destrezas y aspectos afectivos que se pretenden formar en las y los estudiantes al momento de desarrollar la guía de aprendizaje.

La redacción de los objetivos de aprendizaje debe responder a las preguntas: ¿qué? ¿cómo? y ¿para qué? Además, se debe cuidar que tengan una redacción clara, que sean medibles y realizables. Los objetivos también delimitan las acciones metodológicas y los procesos de evaluación.

\section{Producto (s) a desarrollar y forma de entrega:}

Explicar con claridad el producto final que se espera de la guía de aprendizaje, por ejemplo: la entrega de un ensayo con postura crítica, la elaboración de un cuadro sinóptico, la creación de un texto paralelo, el envío de un caso resuelto, el análisis crítico de una teoría, explicar los pasos de un experimento, enviar el reporte de un laboratorio realizado en casa, responder las preguntas abiertas de la guía, resolver las operaciones enviadas, entro otros productos.

Ante la situación que estamos viviendo es prudente pensar en productos o trabajos que sean de fácil entrega por vía electrónica o mediante teléfonos inteligentes. 


\section{Material de apoyo:}

Detallar el material de apoyo que las alumnas y los alumnos necesitarán para el desarrollo de la guía de aprendizaje, por ejemplo: la lectura del capítulo 4 de libro "X", la consulta de dos artículos indexados que han sido enviados por WhatsApp en formato PDF, ver un video corto relacionado con "Y" tema, escuchar un anuncio de radio o verlo en la televisión, escuchar y analizar una conversación, entre otros materiales.

\section{Describir los pasos o procesos:}

En la guía es necesario explicar paso a paso lo que las y los estudiantes deben de realizar para que cumplan con todos los procesos requeridos para su aprendizaje (ver ejemplo al final del documento). [El ejemplo no se comparte en este ensayo].

\section{Fecha de entrega:}

7. Forma de entrega:

\section{Punteo o valoración:}

\section{Rúbrica o criterios de evaluación:}

Detallar todos los aspectos que se van a evaluar para que la y el estudiante tenga claridad sobre qué será evaluado. La evaluación debe responder a los objetivos de aprendizaje antes planteados para que sea formativa.

Es recomendable señalar también el valor o punteo del trabajo o producto a entregar y cómo serán asignados los mismos, además se debe elaborar una rúbrica con los aspectos a evaluar, como se coloca en el ejemplo [el ejemplo no se coloca en este ensayo].

\section{Manual docente para el uso de WhatsApp}

Ya contando con la Guía de aprendizaje asincrónico, de forma simultánea se creó un Manual docente para el uso del WhatsApp como herramienta de apoyo al proceso de aprendizaje (Facultad de Humanidades 2020), el cual contiene orientaciones para utilizar la herramienta desde perspectivas didáctico-pedagógicas, que incluye videos tutoriales para generar las acciones que el docente requiere. La Guía indica que «el propósito principal del WhatsApp es facilitar la comunicación. El reto actual consiste en convertir esta aplicación en un recurso didáctico que potencie el aprendizaje sabiendo que la educación parte de una comunicación efectiva» 
(p. 2). El uso del manual fue incluido en el proceso de capacitación docente antes señalado, con la finalidad de brindar a las profesoras universitarias y a los profesores universitarios dos herramientas de apoyo al proceso de aprendizaje de sus estudiantes con poca conectividad a internet. El Manual docente, que incluye los vínculos a los vídeos, se puede descargar de forma libre y gratuita en: http:// moodlelandivar.url.edu.gt/programas/Manual_WhatsApp_celular.pdf

Es importante resaltar que al momento de elaborar el manual se tenía consciencia que la tecnología es un medio para favorecer los procesos de aprendizaje y que la misma no es un fin en sí misma, lo cual posibilita utilizar diversidad de medios para facilitar procesos de aprendizaje. En este caso, se optó por utilizar dicha herramienta por los resultados mostrados en el proyecto piloto implementado y mencionado antes.

De acuerdo con Calero (2014), el discurso que se comparte a través del WhatsApp y en otras herramientas similares es "híbrido", dado «que comparten elementos propios de los dos canales habituales de comunicación lingüística: el oral y el escrito (gráfico-icónico). Por tal razón, este tipo de lenguajes virtuales han sido denominados textos escritos oralizados» (p. 87), además de caracterizarse por su inmediatez, lo que permite mantener una comunicación virtual cercana o muy similar a la relación oral presencial, al generar confianza por parte de los interlocutores. A ello, debemos añadir que el WhatsApp es «un servicio de mensajería instantánea cuyo propósito es comunicar, vía Internet y en tiempo real, a dos o más usuarios entre sí con mínimos costos económicos... por los mensajes de texto (o archivos de audios, videos, imágenes, o ubicación geográfica) enviados» (p. 90).

El manual goza de diferentes apartados que la lectora y el lector pueden apreciar en el vínculo compartido. Tiene una introducción, aspectos orientadores para el uso de las herramientas propias de la App y los lineamientos de cómo las mismas pueden ser implementadas desde una mirada educativa, además de incluir algunos testimonios de docentes y estudiantes en relación con el uso de dicha herramienta. El manual orienta a las y los docentes sobre aspectos vinculados a responder dudas, hacer tutorías online con baja conectividad, realizar evaluaciones orales, resolver dudas puntuales (grupales o individuales), elaborar discusiones con base en preguntas generadoras, implementar breves encuestas relacionadas con los temas de aprendizaje, sondear conocimientos previos del estudiantado frente a un nuevo tema a desarrollar, crear sentido de pertenencia al grupo en época del confinamiento, mantener un acompañamiento cercano con las alumnas y los alumnos, identificar desventajas del uso de la herramienta, orientar para la creación de grupos de trabajo, acciones para desarrollar competencias educativas, lineamientos para la creación de rúbricas de evaluación, cómo llevar a cabo una clase sincrónica, 
entre otros aspectos que el lector y la lectora podrán encontrar a la hora descargar el archivo en el vínculo ya compartido.

Por su parte, las alumnas y los alumnos han manifestado que el uso de la herramienta, la cual administra la y el docente con base en el manual, les ha favorecido en no abandonar su formación universitaria y en continuar adquiriendo procesos de aprendizaje propios de su formación profesional, tal como lo comenta un estudiante del Campus de Quiché, quien afirma que «el WhatsApp me ha permitido retroalimentación y orientación sobre el curso. El acompañamiento de mi docente ha sido fluido, me ha proporcionado la información por este medio» (Facultad de Humanidades de la Universidad Rafael Landívar, 2020, p. 15).

De acuerdo con Cisco (2010, p. v (El documento utiliza números romanos en las primeras páginas), para lograr el proceso de construcción de una sociedad de aprendizaje

es preciso forjar una nueva coalición que aproveche las innovaciones de todos los sectores de la sociedad, en beneficio de los estudiantes. Esta coalición debe movilizar nuevas estructuras, nuevos enfoques y nueva tecnología para ofrecer un nuevo conjunto de competencias a una población que se dedicará a aprender a lo largo de toda su vida.

aspectos que fueron tomados en cuenta al momento de crear el Manual docente para el uso del WhatsApp como herramienta de apoyo al proceso de aprendizaje, ya que el uso del mismo persigue que las y los estudiantes desarrollen competencias digitales, aunque tengan escasa conectividad, y mantengan relación con la sociedad informatizada.

Dentro de los criterios didáctico-pedagógicos que se tomaron en cuenta para hacer del WhatsApp una herramienta al servicio del aprendizaje, son los elementos señalados por Seas (2016), quien tomando en cuenta los aspectos detallados por Cisco (2010), indica que los procesos educativos propios de la sociedad del aprendizaje vinculados con el uso de las Tic, deben:

Generar una cultura de aprendizaje permanente. Tiene por objeto desarrollar estudiantes motivados y comprometidos que están preparados para responder a los desafíos imprevistos del futuro, así como los del presente. Acerca el aprendizaje al estudiante, puesto que considera el aprendizaje como una actividad, no un lugar físico. Considera que el aprendizaje es para todos y que nadie debe quedar excluido. Reconoce que las personas aprenden de maneras diferentes y procura satisfacer esas necesidades. Cultiva e integra nuevos proveedores educativos, de los sectores 
público, privado y sin fines de lucro. Desarrolla nuevas relaciones y redes entre los estudiantes... Sustenta sistemas de innovación y retroalimentación continuas para conocer qué cosas funcionan y en qué circunstancias. (p. 302)

Lo cual ha garantizado que el Manual docente no sea únicamente un medio de comunicación informático o una serie de chats sino una herramienta educativa que administra la educadora y el educador para logar el aprendizaje de sus estudiantes desde el uso del teléfono celular.

\section{Conclusión}

Finalmente, el compartir estas dos herramientas elaboradas para desarrollar procesos asincrónicos que garanticen el aprendizaje autónomo de las y los estudiantes demuestra que es posible generar acciones educativas diferentes, que no dependan en su totalidad de la conexión informática, de modo que las y los alumnos con poco acceso digital puedan continuar con sus procesos de aprendizaje en el nivel superior.

En sintonía con la anterior afirmación, Audrey Azoulay (2020, p. 1), directora general de Unesco, indica que «la educación es un derecho humano fundamental que debemos asegurar y defender todos los días, especialmente teniendo en cuenta las nuevas y graves formas de exclusión, sobre todo en la esfera digital», tema que ha sido expuesto al inicio de este texto, con el deseo permanente de proteger el derecho a la educación y promover la igualdad de oportunidades de aprendizaje sin distinción de raza, género, edad, condiciones socioeconómicas, pertenencia cultural, ubicación geográfica, acceso a recursos informáticos o cualquier otra distinción.

Estos aspectos también inspiraron la elaboración del material aquí presentado, ya que la pandemia causada por la COVID-19 ha potencializado las desigualdades preexistentes en todo el mundo y ha aumentado la probabilidad de que las y los estudiantes vulnerables se queden atrás en sus procesos de aprendizaje, sobre todo en países en donde los Estados no garantizan la inclusión de las más marginadas y los más marginados y, por tanto, las educadoras y los educadores deben ser capaces de responder a dichos desafíos.

Queda demostrado así, que es posible dar continuidad a los procesos de aprendizaje de las y los estudiantes universitarios con poco acceso a internet desde las herramientas presentadas en este ensayo, de modo que de forma permanente las instituciones educativas busquen soluciones creativas, innovadoras y viables que garanticen el derecho humano a la educación más allá de las dificultades que nos presenta la brecha digital actual. 


\section{Referencias}

Agencia EFE. (2019). Solo el 29,3 \% de la población censada en Guatemala utiliza internet. Publicación del 20 de septiembre. Guatemala: EFE. https:/www.efe.com/ efe/america/tecnologia/solo-el-29-3-de-la-poblacion-censada-en-guatemala-utiliza-internet/20000036-4068618\#: :text=El\%20censo\%20alcanz\%C3\%B3\%20a\%20 m\%C3\%A1s,12\%2C5\%20millones\%20de \%20personas.

Azoulay, A. (2020). Poner fin a la discriminación en la educación: un instrumento clave para proteger el derecho a la educación. Unesco. https://es.unesco.org/news/ poner-fin-discriminacion-educacion-instrumento-clave-proteger-derecho-educacion

Calero, M. (2014). El discurso del WhatsApp: entre el Messenger y el SMS. Revista ORALIA, 17, 85-114. https://www.academia.edu/9403334/ El_discurso_del_WhatsApp_entre_el_Messenger_y_el_SMS

Cisco. (2010). La sociedad del aprendizaje. Networking Academy.https://www.cisco.com/c/ dam/en_us/about/citizenship/socio-economic/docs/TLS_Spanish.pdf

Departamento de Educación, Facultad de Humanidades, Universidad Rafael Landívar de Guatemala. (2020). Elaboración de guías para el desarrollo del aprendizaje autónomo y uso de WhatsApp como herramienta de apoyo educativo. Docentes en acción. Folleto de capacitación. Guatemala: Universidad Rafael Landívar.

Escobar-Galo, J. y Ambrosy, I. (2000). Guía de actividad de aprendizaje asincrónico o a distancia. Material para capacitación docente. Guatemala: Universidad Rafael Landívar de Guatemala.

Facultad de Humanidades. (2020). WhatsApp Herramienta de apoyo al proceso de aprendizaje, manual docente. Material de apoyo didáctico-pedagógico. Guatemala: Universidad Rafael Landívar. http://moodlelandivar.url.edu.gt/programas/Manual_WhatsApp_celular.pdf https://ceat.url.edu.gt/pagina/whatsapp/

Freire, P. (2008). El grito manso. 2. a edición. Argentina: Siglo veintiuno revisores.

Giannini, S. y Grant, S. (2020). Tres formas de planificar la equidad durante los cierres de escuelas por coronavirus. Unesco. http://www.iiep.unesco.org/en/ three-ways-plan-equity-during-coronavirus-school-closures-13365

Instituto Nacional de Estadística de Guatemala. (2018). XII Censo nacional de población y vivienda. https://www.censopoblacion.gt/comovivimos

Seas, J. (2016). Didáctica General I. Costa Rica: Editorial Universidad Estatal a Distancia. 
